\title{
A Best Known Method to Effectively Differentiate Elements with XEDS Peaks Overlapping for High-Volume Manufacturing of Semiconductor Device at Wafer Foundries
}

Wayne Zhao

\section{GLOBALFOUNDRIES, Malta, New York, United States}

One theme of efforts in high-volume manufacturing (HVM) of semiconductor Integrated Chips (ICs) at Si-based Wafer-Foundries is to constantly improve yield percentage by reducing inline defects. This is especially critical with the continuous miniaturization of semiconductor device with nanometer transistors as the basic building block [1 3]. Therefore, one key task for a Physical Failure Analysis (PFA) team at a wafer foundry is to QUICKLY identify inline defect source and provide meaningful feedback to the process and integration teams, so that effective mitigations can be immediately applied to ensure the production line healthy. To pin-point the defect source at the quickest turnaround time (TAT) by PFA is vital to the business success at a Wafer-foundry.

Semiconductor integration is an extremely complex materials system, which involves over a thousand steps of various processes. Roughly, defect sources can be grouped into the following categories. Listed here are just examples only, not necessarily comprehensive, since each technology node has different integration schemes. First, from the materials that eventually have been built into the semiconductor device, based on IC industry standards pertinent to each technology node, such as various phases of $\mathrm{Si}$, $\mathrm{O}, \mathrm{N}, \mathrm{C}, \mathrm{Ti}, \mathrm{Hf}, \mathrm{Al}, \mathrm{Ge}, \mathrm{P}, \mathrm{As}, \mathrm{B}, \mathrm{W}, \mathrm{Ni}, \mathrm{Co}, \mathrm{Cu}, \mathrm{Ta}, \mathrm{Mn}$, and/or rare earth elements for special needs, etc. Second, materials for process only, but should not remain in the IC end product, such as $\mathrm{HF}, \mathrm{HBr}$, Ar, TiCl4, spin-on hardmask (SOH), photoresist, anti-reflective coatings (ARC), Ce-oxide from chemical-mechanical planarization / polishing (CMP) slurry, etc, Third, various materials from processing chambers for these semiconductor IC wafers, depending on vendors' choice, such as stainless steels, ceramics, and coatings, e.g., $\mathrm{Fe}, \mathrm{C}, \mathrm{O}, \mathrm{Mo}, \mathrm{Ni}, \mathrm{Co} \mathrm{Cr}, \mathrm{Y}, \mathrm{Ba}, \mathrm{Mg}, \mathrm{Ag}$, etc. Last, materials from PFA lab during delayering and cross-section defects, such as, $\mathrm{C}, \mathrm{Pt}, \mathrm{Mo}, \mathrm{Cu}, \mathrm{Al}, \mathrm{Zr}, \mathrm{Ga}$, Ar, etc. Plus elements from components either inside focus ion beam (FIB) or various electron microscopes, which can be detected by the Analytical Transmission Electron Microscopy (Analytical TEM or AEM) elemental analysis components, such as, the X-ray Energy Dispersive Spectroscopy (XEDS) and/or the Electron Energy Loss Spectroscopy (EELS). To emphasize, for any inline excursion, source of defects to be sorted out could be either systematically induced by a particular category or randomly from any combination of few or even most of these categories aforementioned.

With such a bizarre / complex materials system, especially when certain elements having strong overlapping peaks in XEDS and/or EELS spectra, to differentiate effectively these elements with various combinations at a quickest possible turnaround time is a BIG challenge to PFA teams at wafer foundries. No single best known method (BKM) can be universally applied to address the challenges. More flexible approaches have to be taken to effectively differentiate them, and a lot of hands-on experience in the semiconductor industry is a great value.

Reported here, Figs-1a 1e, is one example to quickly effectively differentiate $\mathrm{Ba}, \mathrm{Ce}, \mathrm{Ti}$, and $\mathrm{Cr}$, by extending XEDS detection energy range from the default $20 \mathrm{KeV}$ to $40 \mathrm{KeV}$. This approach facilitated the display of XEDS peaks from the whole family of given elements of interest, such as $\mathrm{Ba}$, and $\mathrm{Ce}$. Major peaks of $\mathrm{Ba}$ and $\mathrm{Ce}$ in the XEDS spectra can be scrutinized and less/or even no overlapping XEDS peaks can be employed for quantification of the XEDS mapping. This helped to differentiate / 
clarify the ambiguity to the greatest extent with a high-level of confidence. Sounds like a simple trick, but this novel approach means a lot at wafer foundries in terms of business impact, since time is money at wafer-fabs. This BKM not only works great for IC industry PFA purpose with a simple and quickest TAT, but can serve as a good educational guideline for beginners who have an interest and desire to pursue PFA and materials analysis as a career. For TEM with 200 300KV acceleration voltage, this XEDS detection range can be expanded to $80 \mathrm{KeV}$, but for labs still using old XEDS model only a $20 \mathrm{KeV}$ setting, alternative method such as EELS has to be employed, which will cost more time to respond. Other applications of this BKM can be to differentiate Ag and Ar, not shown here, due to page limit.

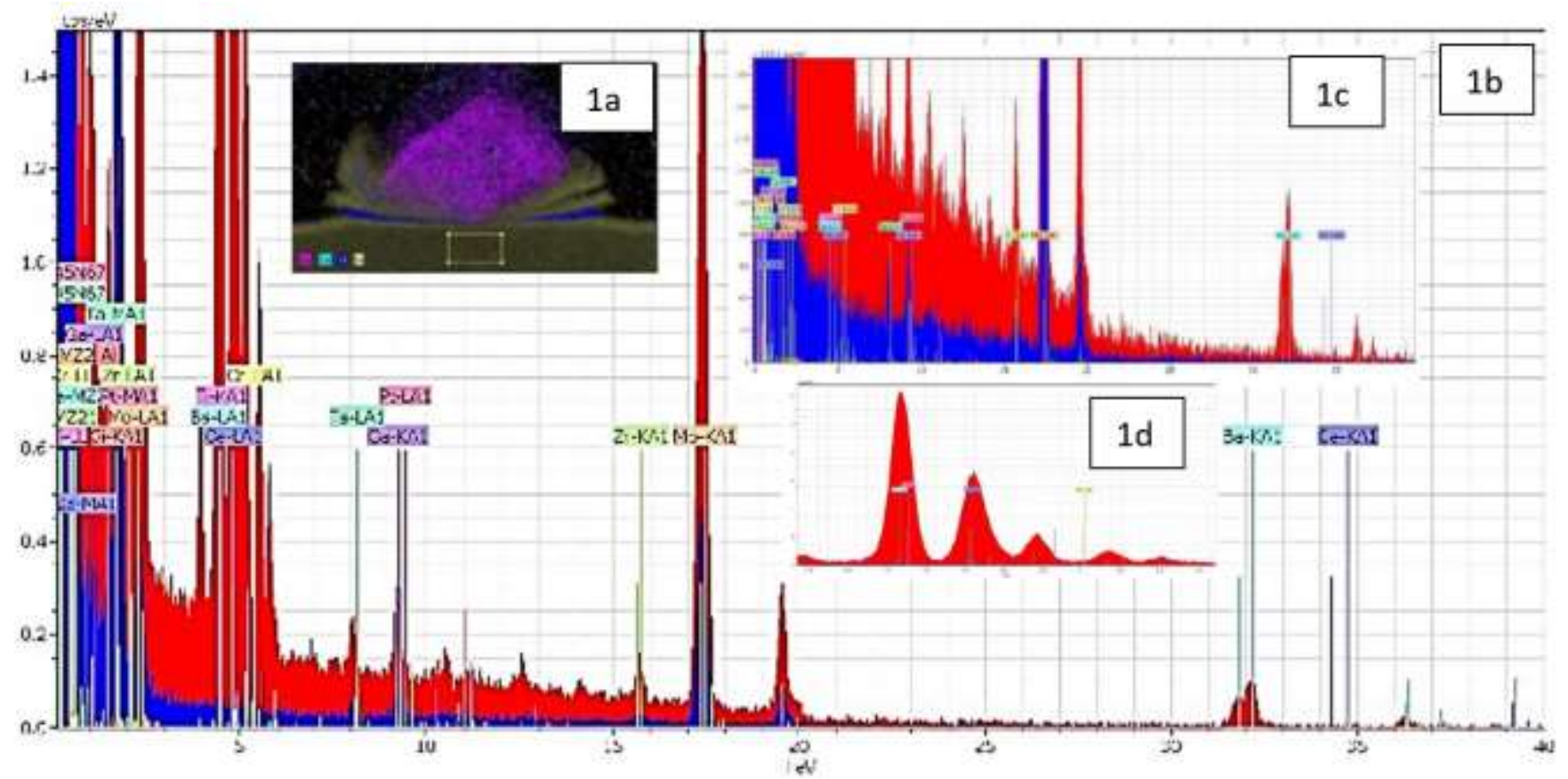

Figure 1. (a) an overlay of XEDS mapping of $\mathrm{Ba}, \mathrm{Ce}, \mathrm{Ti}$ and $\mathrm{Si}$ in the defect particle; and (b) (d) X-ray EDS spectra of $40 \mathrm{Kev}$ and vertical (c) and horizontal (d) zoomed-in views. Red spectrum from the particle, and the blue spectrum from the known $\mathrm{Si}+\mathrm{O}$ layer as a reference.

\section{References}

[1] S. Siddiqui, R. Galatage, W. Zhao, et al., Microelectronic Engineering, Volume 223, (2020), p. 111219.

[2] M. A. Gribelyuk, B. Fu, W. Zhao, Journal of Applied Physics, 125, (2019); pp. 165306

[3] W. Zhao and Y. Y. Wang,Microscopy \& Microanalysis, Vol. 21 (Supplement 1), (2017), pp.1490 1491.

[4] Thanks to Irene Brooks for proof-reading, Gerald Walker for his skillfulness in TEM-prep and Long Men for screening the TEM sample; and Fab8 Management and Legal teams for supporting the publication clearance. 